\title{
23. ON THE DIFFERENTIAL CORRECTION OF NEARLY PARABOLIC ORBITS
}

\author{
P. HERGET \\ University of Cincinnati Observatory, Cincinnati, Ohio, U.S.A.
}

The differential correction of nearly parabolic orbits was discussed by the author (Herget, 1939) in the era of lead pencil computing. The Gauss-Marth method is the best one to use whenever the appropriate conditions exist, i.e., $|E|<64^{\circ}$ and $e$ nearly unity. The crucial point in the above-cited discussion is the use of the first differences from the Gauss-Marth tables in order to simplify the computation of the partial differential coefficients, namely $\mathrm{d} B / \mathrm{d} A, \mathrm{~d} C / \mathrm{d} A$, and $\mathrm{d} D / \mathrm{d} A$.

In the present era of computation by electronic calculators, the Gauss-Marth tables have been replaced by series expansions (Benima et al., 1969), which are more appropriate to the use of high-speed electronic storage devices. The purpose of this note is simply to call attention to the efficacy of a subroutine which will evaluate the derivatives of these series, and then the same formulae may be used for the partial differential coefficients as were cited above. The arrangement of formulae in the form of matrix multiplications is still a useful device in modern programming methods.

At the Cincinnati Observatory we have attained what is probably the ultimate in the differential correction of orbits by expanding the widely used $N$-body integration program of Schubart and Stumpff (1966). The partial differential coefficients are each obtained by a separate, implicit, numerical double integration in exactly the same way as was presented by the writer (Herget, 1968) for the outer satellites of Jupiter. This is equivalent to the simultaneous integration of the trajectory and its variational equation, and this leaves little more to be desired, especially if there are excessive perturbations involved. This program has been used with great success in our study of the motion of P/Pons-Brooks during two complete revolutions (Herget and Carr, 1972).

\section{References}

Benima, B., Cherniack, J. R., Marsden, B. G., and Porter, J. G. : 1969, Publ. Astron. Soc. Pacific 81, 121.

Herget, P.: 1939, Astron. J. 48, 105.

Herget, P.: 1968, Astron. J. 73, 737.

Herget, P. and Carr, H. J.: 1972, this Symposium, p. 195.

Schubart, J. and Stumpff, P.: 1966, Veroeffentl. Astron. Rechen-Inst. Heidelberg No. 18. 